\title{
An efficient global optimization algorithm based on augmented radial basis function ${ }^{\star}$
}

\author{
Yun-Kang Sui ${ }^{1, a}$, Shan-Po $\mathrm{Li}^{1}$ and Ying-Qiao Guo ${ }^{2}$ \\ 1 Centre of Numerical Simulation for Engineering, Beijing University of Technology, F100022 Beijing, P.R. China \\ 2 Laboratory of Mechanics, Materials \& Structures, University of Reims Champagne-Ardenne, 51687 Reims, France
}

Received 29 August 2007, accepted 18 February 2008

\begin{abstract}
In the structural optimization, the accuracy of approximation for the established mathematical model will directly affect the solution efficiency, even the convergence. The global optimization model based on the augmented Gaussian radial basis function $h$ as a high approximation accuracy, but the solution efficiency will not be increased without a matched optimization algorithm. In this paper, we adopt the information at the interpolating points in large extent and the augmented Gaussian radial basis function to construct the approximate mathematical model. Using the explicit derivatives of the model for the sensitivities and sequential quadratic programming (SQP) algorithm for the optimization solving, an efficient algorithm of global optimization is proposed. It is simple to be realized and converges quickly. Two examples will illustrate the stability and efficiency of the present algorithm.
\end{abstract}

Key words: Structural optimization; approximate model; Gaussian radial basis function; global optimization algorithm

\section{Introduction}

In 1960, Schmit [1] brought forward the idea which introduced the mathematical programming into the structural optimization by using the systematic synthesis. At that time, he might not realize the importance of the establishment of mathematical models, so the optimal design was found by hundreds structural reanalyses. In fact, the objective function and constraint functions in an engineering problem can be rarely written as the explicit expressions in terms of design variables, the sensitivity analysis is very time-consuming.

In 1974, Schmit and Farshi [2] proposed some approximation concepts including the obtaintion of explicit approximate functions for constraint functions to improve the structural optimization efficiency and the iteration times largely decreased. Essentially, the establishment of an optimization model consists in constructing approximate functions for objective function or/and constraint conditions. From then on, the idea of establishing mathematical model has been accepted by many optimization researchers and is becoming a relative independent research domain. However, some researchers still use the methods without establishing any optimization

\footnotetext{
* Supported by National Natural Science Foundation of China (10472003) and Scientific Research Foundation of Doctoral Subjects in Chinese Universities (20060005010).

a Corresponding authors:

ysui@bjut.edu.cn; 1sp102@emails.bjut.edu.cn;

yq.guo@univ-reims.fr
}

model. Although the difficulty of sensitivity analysis can be avoided, the calculations are very time-consuming and the problem of converge often arises.

Therefore, the establishment of an approximate optimization model is a good choice for the structural optimization. The research works on the model establishment can be divided into three parts: the first one is to select suitable approximate functions, the second one is to provide the derivatives of these functions (sensitivity analysis), the third one is to choose an adapted algorithm to solve the optimization model. In the early used methods for mathematic model establishing, the Taylor's expansions of first order and/or second order are usually used, and analytical methods of sensitivity analysis are often adopted. The disadvantages of the sensitivity analysis are that the analytical derivatives is very difficult and the computation of derivatives is too expensive. This may be the reason that some scholars do not establish any optimization model.

Are there some methods to establish the mathematical model without sensitivity analysis? Recently, the Response Surface Methodology (RSM) (Mayer et al. [3]) is largely used for the structural optimization, a lot of research (Venter and Haftka [4], Hosder et al. [5], Jansson et al. [6], Liu et al. [7]) and improved works (Zheng and Das [8], Sui and Li [9]) have been done by using the RSM. The RSM is mainly a polynomial regression method, it usually uses the first order and/or second order polynomials and the approximation accuracy is good only in local domain. It is often combined with QP algorithm by 
sequential iterations, so an initial solution should be selected by user. Because this selection is very difficult in a structural optimization, the method can not promise to give the global optimal solution. Although high order polynomials can be used to obtain some global approximations, but a lot of response calculations are needed at the experiment points and this will lead to too expensive calculations.

In recent years, some researchers introduced an accurate global approximate method called Radial Basis Function method (RBF) (Mason and Cox [10], Krishnamurthy [11]). Fang and Horstemeyer [12] demonstrate that the approximation accuracy of the augmented Gaussian RBF is higher than that of other types of RBF and its approximation domain is also wider. How to solve this type of models with high approximation accuracy? From published papers, an optimization algorithm is often taken to solve the model without considering whether the algorithm is adapted to the characteristic of the model. In other word, it is deficient in considering the relation between an establishing approximate optimization model and an adapted algorithm. Consequently, the optimization computation is not efficient. In this paper, we introduce a new sequential algorithm corresponding to the approximation model based on the augmented RBF method. The numerical results illustrate the accuracy and efficiency of the present algorithm.

\section{RBF and augmented RBF methods}

The Radial Basis Function (RBF) can be expressed by the linear combination of the functions in terms of radial distance from the considered point to every interpolation point (or sample point or experiment design point) [12]:

$$
s(\mathbf{x})=\sum_{j=1}^{m} \lambda_{j} \varphi\left(\left\|\mathbf{x}-\mathbf{x}^{j}\right\|_{2}\right) \quad \mathbf{x} \in E^{n}
$$

where $n$ is the total number of design variables or the dimensional number of the design space, $m$ is the total number of interpolating points, $\lambda_{j}(j=1, \ldots, m)$ is the $j$ th coefficient to be determined, $\mathbf{x}$ is the position vector of a design point, $\mathbf{x}^{j}$ is the position vector of $j$ th interpolating point, $\left\|\mathbf{x}-\mathbf{x}^{j}\right\|_{2}$ is the $j$ th radial distance between the two points:

$$
r^{0 j}=\left\|\mathbf{x}-\mathbf{x}^{j}\right\|_{2}=\sqrt{\sum_{k=1}^{n}\left(x_{k}-x_{k}^{j}\right)^{2}} .
$$

Different forms of the $\operatorname{RBF} \varphi(r)$ are listed in Table 1.

Let $F_{i}$ the real response value at the interpolating point $\mathbf{x}^{i}$, the formula (1) becomes:

$$
\begin{gathered}
\sum_{j=1}^{m} \lambda_{j} \varphi\left(\boldsymbol{r}^{i j}\right)=F_{i} \quad(i=1, \ldots, m) \\
r^{i j}=\left\|\mathbf{x}^{i}-\mathbf{x}^{j}\right\|_{2}=\sqrt{\sum_{k=1}^{n}\left(x_{k}^{i}-x_{k}^{j}\right)^{2}} .
\end{gathered}
$$

Table 1. Usually used radial basis functions.

\begin{tabular}{cc}
\hline Function name & Expression \\
\hline Linear & $\phi(r)=c r$ \\
Thrice & $\phi(r)=c r^{3}$ \\
Thin plate spline & $\phi(r)=r^{2} \ln (c r), 0<c \leqslant 1$ \\
Gaussian & $\phi(r)=e^{-c r^{2}}, 0<c \leqslant 1$ \\
Multi-quadratic & $\phi(r)=\sqrt{r^{2}+c^{2}}, 0<c \leqslant 1$ \\
\hline
\end{tabular}

where the constant $c$ is usually taken as 1 .

Then $\lambda_{j}(j=1, \ldots, m)$ can be obtained by solving equations $(3)$.

To increase the approximation accuracy and expand the approximation range of the RBF, a polynomial term can be added to the expression (1):

$$
s(\mathbf{x})=\sum_{j=1}^{m} \lambda_{j} \varphi\left(\mathbf{r}^{0 j}\right)+\sum_{i=1}^{I} b_{i} P_{i}(\mathbf{x})
$$

where the polynomial functions $p_{i}(\mathbf{x})(i=1, \ldots, I)$ can be $1, x_{1}, \ldots, x_{n}, x_{1}^{2}, x_{1} x_{2}, \ldots, x_{n}^{2}, \ldots$ or a part of them. The above expression is called augmented RBF. Using $m$ interpolating points and their corresponding real response values in expression (5), we obtain:

$$
\left\{\begin{array}{c}
\lambda_{1} 0+\lambda_{2} \varphi\left(\mathbf{r}^{12}\right)+\ldots+ \\
+\lambda_{m} \varphi\left(\mathbf{r}^{1 m}\right)+b_{1} p_{1}\left(\mathbf{x}^{1}\right)+\ldots \\
\quad+b_{I} p_{I}\left(\mathbf{x}^{1}\right)=F_{1} \\
\lambda_{1} \varphi\left(\mathbf{r}^{21}\right)+\lambda_{2} 0+\ldots+\lambda_{m} \varphi\left(\mathbf{r}^{2 m}\right)+b_{1} p_{1}\left(\mathbf{x}^{2}\right)+\ldots \\
+b_{I} p_{I}\left(\mathbf{x}^{2}\right)=F_{2} \\
\vdots \\
\lambda_{1} \varphi\left(\mathbf{r}^{m 1}\right)+\lambda_{2} \varphi\left(\mathbf{r}^{m 2}\right)+\ldots+\lambda_{m} 0+b_{1} p_{1}\left(\mathbf{x}^{m}\right)+\ldots \\
+b_{I} p_{I}\left(\mathbf{x}^{m}\right)=F_{m}
\end{array}\right.
$$

In equations $(6)$, there are $m$ coefficients $\lambda_{j}(j=1, \ldots, m)$ and $I$ coefficients $b_{i}(i=1, \ldots, I)$ as unknowns, so $I$ equations have to be added. The added equations may be obtained according to the following orthogonal conditions:

$$
\left\{\begin{array}{c}
p_{1}\left(\mathbf{x}^{1}\right) \lambda_{1}+\ldots+p_{1}\left(\mathbf{x}^{m}\right) \lambda_{m}=0 \\
\vdots \\
p_{I}\left(\mathbf{x}^{1}\right) \lambda_{1}+\ldots+p_{I}\left(\mathbf{x}^{m}\right) \lambda_{m}=0 .
\end{array}\right.
$$

The equations (6) and (7) can be rewritten in a matrix form:

$$
\begin{aligned}
{\left[\begin{array}{ll}
\mathbf{A} & \mathbf{B} \\
\mathbf{B}^{\mathrm{T}} & \mathbf{0}
\end{array}\right]\left\{\begin{array}{l}
\lambda \\
\mathbf{b}
\end{array}\right\}=\left\{\begin{array}{l}
\mathbf{F}\} \\
\mathbf{0}
\end{array}\right\} } \\
\text { with } \mathbf{A}=\left[\begin{array}{cccc}
0 & \varphi\left(\mathbf{r}^{12}\right) & \ldots & \varphi\left(\mathbf{r}^{1 m}\right) \\
\varphi\left(\mathbf{r}^{21}\right) & 0 & \ldots & \varphi\left(\mathbf{r}^{2 m}\right) \\
\vdots & \vdots & \vdots & \vdots \\
\varphi\left(\mathbf{r}^{m 1}\right) & \varphi\left(\mathbf{r}^{m 2}\right) & \ldots & 0
\end{array}\right], \\
\mathbf{B}=\left[\begin{array}{ccc}
p_{1}\left(\mathbf{x}^{1}\right) & \ldots & p_{I}\left(\mathbf{x}^{1}\right) \\
\vdots & \vdots & \vdots \\
p_{1}\left(\mathbf{x}^{m}\right) & \ldots & p_{I}\left(\mathbf{x}^{m}\right)
\end{array}\right]
\end{aligned}
$$




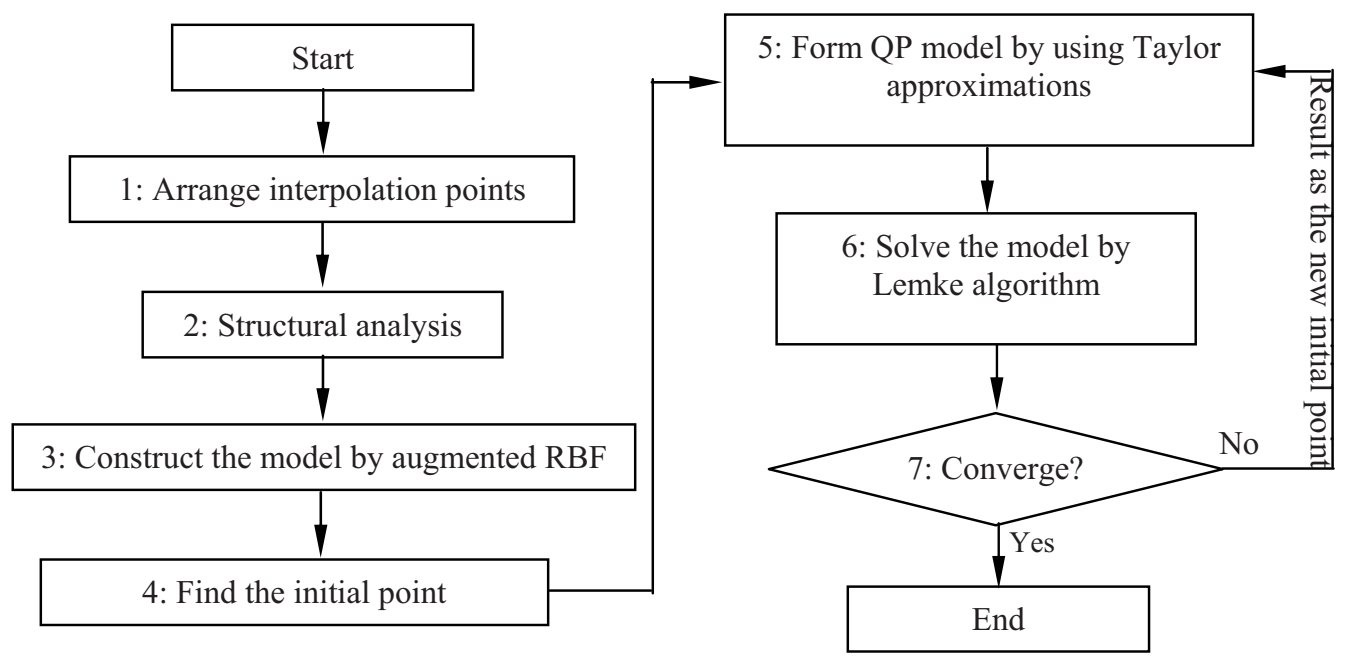

Fig. 1. Flow chart of present new algorithm.

$\lambda=\left(\lambda_{1}, \ldots, \lambda_{m}\right)^{\mathrm{T}}, \mathbf{b}=\left(b_{1}, \ldots, b_{I}\right)^{\mathrm{T}}, \mathbf{F}=\left(F_{1}, \ldots, F_{m}\right)^{\mathrm{T}}$.

The RBF and augmented RBF have no error at the interpolating points. In order to evaluate their approximation accuracy, $K$ other points are selected in the design space. We calculate the approximate response $\bar{F}_{i}(i=1, \ldots, K)$ using (5) and the real response $F_{i}^{r e a l}(i=1, \ldots, K)$ using the structural analysis solver, then the following root of mean square errors can be the evaluate index:

$$
R M S E=\sqrt{\sum_{i=1}^{K}\left(\overline{F_{i}}-F_{i}^{\mathrm{real}}\right)^{2} / K}
$$

\section{Implementation of algorithms of modelling and optimization}

The optimization problem of a mathematical programming or a structural optimization can be described by:

$$
\begin{cases}\text { find } & \mathbf{x} \in E^{n} \\ \min & f(\mathbf{x}) \\ \text { s.t. } & c_{k}(\mathbf{x}) \leqslant b_{k}(k=1, \ldots, K) \\ & \underline{x}_{i} \leqslant x_{i} \leqslant \bar{x}_{i}(i=1, \ldots, n)\end{cases}
$$

where $K$ is the total number of behaviour constraints. The above mentioned Augmented RBF (Eq. (5)) can be used to approach the objective function and constraints functions in order to obtain an optimization model in global domain. But it is difficult to solve this mathematic model because of its high nonlinearity.

In order to find an efficient optimization algorithm matched with high order nonlinear characteristics of the model (9), the following solving strategy including the sequential quadratic programming $(\mathrm{SQP})$ is proposed. It is carried out in 7 steps:

(1) Arrange a set of interpolating points evenly in the global domain representing the experiment design variables. Their feasible properties are not considered.
(2) A series of structural analysis at the interpolating points are carried out to obtain the corresponding response values.

(3) The objective function and constraint conditions based on the Augment Gaussian RBF are established and their corresponding coefficients $\lambda_{j}$ and $b_{i}$ are determined according to the response values.

(4) The vector of design variables giving the minimal value of the objective function at one of all feasible interpolating points is taken as an initial point for SQP.

(5) At the initial point, a standard QP model is formed by using the second order Taylor's expansion for the objective function and the first order Taylor's expansions for the constraint conditions.

(6) Combined with the rational move limits of design variables, the QP model is solved by Lemke algorithm.

(7) Checking the convergence criterion, if it is satisfied then exit, otherwise return to the 5th step and use the obtained result as a new initial point for the next step.

In 5th step, for the solution convenience, the objective function and the constraint conditions adopt different order Taylor's expansions (Sui et al. [13]). In 6th step, the rational move limits of design variables is a method considering accumulated information (Sui et al. [14]) and Lemke algorithm can efficiently solve a standard QP.

The flow chart in Figure 1 shows our new algorithm, named Augmented Gaussian RBF-SQP (AG-RBF-SQP) method.

In the QP model, the first order and second order Taylor's approximations of an arbitrary function $f(\mathbf{x})$ are given by the following expressions

$$
f(\mathbf{x}) \approx f\left(\mathbf{x}^{0}\right)-\sum_{i=1}^{n} \frac{\partial f\left(\mathbf{x}^{0}\right)}{\partial x_{i}} x_{i}^{0}+\sum_{i=1}^{n} \frac{\partial f\left(\mathbf{x}^{0}\right)}{\partial x_{i}} x_{i}
$$




$$
\begin{aligned}
f(\mathbf{x}) \approx & f\left(\mathbf{x}^{0}\right)-\sum_{i=1}^{n} \frac{\partial f\left(\mathbf{x}^{0}\right)}{\partial x_{i}} x_{i}^{0} \\
& +\frac{1}{2} \sum_{j=1}^{n} \sum_{k=1}^{n} \frac{\partial^{2} f\left(\mathbf{x}^{0}\right)}{\partial x_{j} \partial x_{k}} x_{j}^{0} x_{k}^{0}+\sum_{i=1}^{n}\left(\frac{\partial f\left(\mathbf{x}^{0}\right)}{\partial x_{i}}\right. \\
& \left.-\sum_{j=1}^{n} \frac{\partial^{2} f\left(\mathbf{x}^{0}\right)}{\partial x_{i} \partial x_{j}} x_{j}^{0}\right) x_{i}+\frac{1}{2} \sum_{j=1}^{n} \sum_{k=1}^{n} \frac{\partial^{2} f\left(\mathbf{x}^{0}\right)}{\partial x_{j} \partial x_{k}} x_{j} x_{k} .
\end{aligned}
$$

For our algorithm, the Gaussian functions and linear functions are taken in the formula (5). In order to improve the computation efficiency, the analytical explicit derivatives are deduced. The partial derivatives of first order and second order are calculated as follows:

$$
\begin{gathered}
\partial s(\mathbf{x}) / \partial x_{l}=-2 \sum_{j=1}^{m} \lambda_{j} e^{-\sum_{k=1}^{n}\left(x_{k}-x_{k}^{j}\right)^{2}}\left(x_{l}-x_{l}^{j}\right)+b_{l} \\
\frac{\partial^{2} s(\mathbf{x})}{\partial x_{l} \partial x_{p}}=\left\{\begin{array}{c}
4 \sum_{j=1}^{m} \lambda_{j} e^{-\sum_{k=1}^{n}\left(x_{k}-x_{k}^{j}\right)^{2}}\left(x_{l}-x_{l}^{j}\right)\left(x_{p}-x_{p}^{j}\right) \\
\text { if } l \neq p \\
2 \sum_{j=1}^{m} \lambda_{j} e^{-\sum_{k=1}^{n}\left(x_{k}-x_{k}^{j}\right)^{2}}\left[2\left(x_{l}-x_{l}^{j}\right)^{2}-1\right] \\
\text { if } l=p .
\end{array}\right.
\end{gathered}
$$

Finally, the model (9) can be transformed to a standard QP model:

$$
\begin{cases}\text { find } & \mathbf{x}^{(v)} \in E^{n} \\ \min & \mathbf{x}^{\mathrm{T}} \mathbf{H}^{(v)} \mathbf{x} / 2+\mathbf{C}^{(v)} \mathbf{x} \\ \text { s.t. } & \mathbf{A}^{(v)} \mathbf{x} \leqslant \mathbf{B}-\mathbf{A}_{0}^{(v)} \\ & \underline{x}_{i}^{(v)} \leqslant x_{i} \leqslant \bar{x}_{i}^{(v)}\end{cases}
$$

where $v$ denotes the $v$ th iteration. The objective function is expanded into the second order Taylor expansion: $\mathbf{H}^{(v)}$ is the Hessian matrix, given by the fifth term in the expression (11), $\mathbf{C}^{(v)}$ is a vector, given by the fourth term in (11). The first three terms in (11) have no influence on the minimization of $f(\mathbf{x})$. The constraint functions in (9) are expanded into the first order Taylor expansion: $\mathbf{A}^{(v)}$ is a matrix given by the third term in $(10), \mathbf{A}_{0}^{(v)}$ is a vector given by the first two terms in (10), $\mathbf{B}$ represents $b_{k}$ in (9).

\section{Numerical examples}

In order to evaluate the accuracy and efficiency of the present algorithm, a lot of mathematical functions and practical engineering examples have been treated, we just present two of them in this paper: a mathematical example, an engineering application, whose models are established by the Augmented Gaussian RBF and solved by the present algorithm. The optimal results of the first example is compared with the results obtained by using the

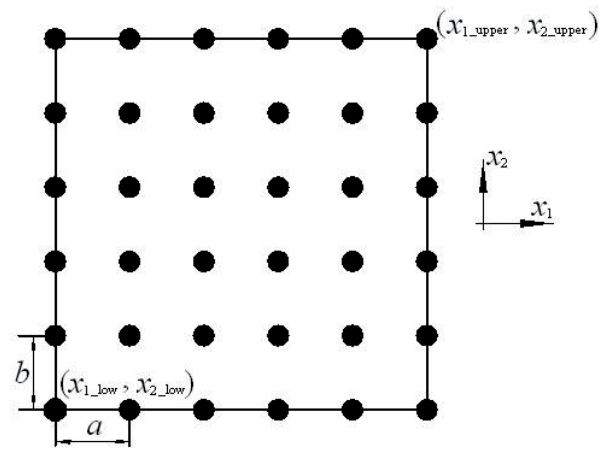

Fig. 2. 36 Interpolating points.

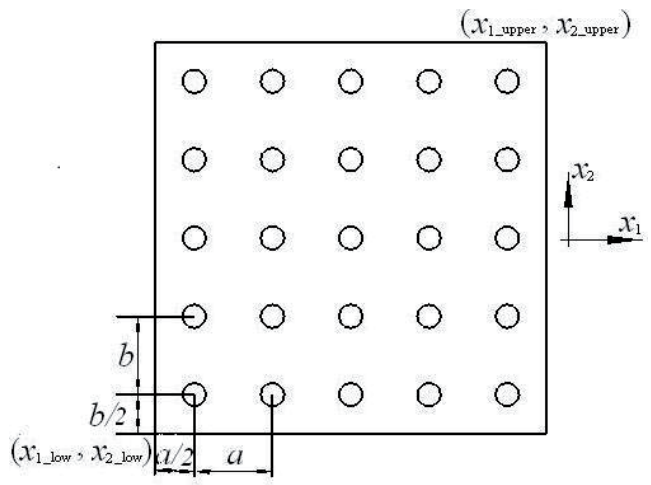

Fig. 3. 25 Non- interpolating points.

Genetic Algorithm (GA) (Xuan et al. [15]) and Simulated Annealing Algorithm (SAA) (Yang and Gu [16]), implemented by the 7D-Soft High Technology Inc. and published in the software 1stOpt1.5 (Website [17]). The optimal results of the second example is compared with that of SRSM-QP (sequential response surface methodology and quadratic programming) algorithm (Yang et al. [18]).

\subsection{Maximization problem of a highly nonlinear objective function}

$$
\begin{cases}\text { find } & \mathbf{x} \in E^{2} \\ \max & f(\mathbf{x}) \\ \text { s.t. } & 0.01 \leqslant x_{1} \leqslant 1 \\ & 0 \leqslant x_{2} \leqslant 1\end{cases}
$$

with $f(\mathbf{x})=\left[0.8 \sqrt{x_{1}^{2}+x_{2}^{2}}+0.35 \sin \left(2.4 \pi \sqrt{x_{1}^{2}+x_{2}^{2}}\right) / \sqrt{2}\right]$ $\left[1.5 \sin \left(1.3 \arctan \left(x_{2} / x_{1}\right)\right)\right]$. The real response surface is illustrated in Figure 4. The exact solution gives the maximum value 1.6519 at the point $(0.4276,1.0)$. To establish an approximate model and evaluate the error, we evenly arrange 36 interpolating points and 25 noninterpolate points respectively in the global design space (Figs. 2 and $3, a=0.198, b=0.2$ ). The approximate model obtained by using the Augmented Gaussian RBF and the given 36 sample points gives an approximate response surface (Fig. 5). The RMSE using the augmented $\mathrm{RBF}$ at the given 25 points is 0.0108 and that using RBF at the given 25 points is 0.0112 . Table 2 gives the comparison of the results of different algorithms. 


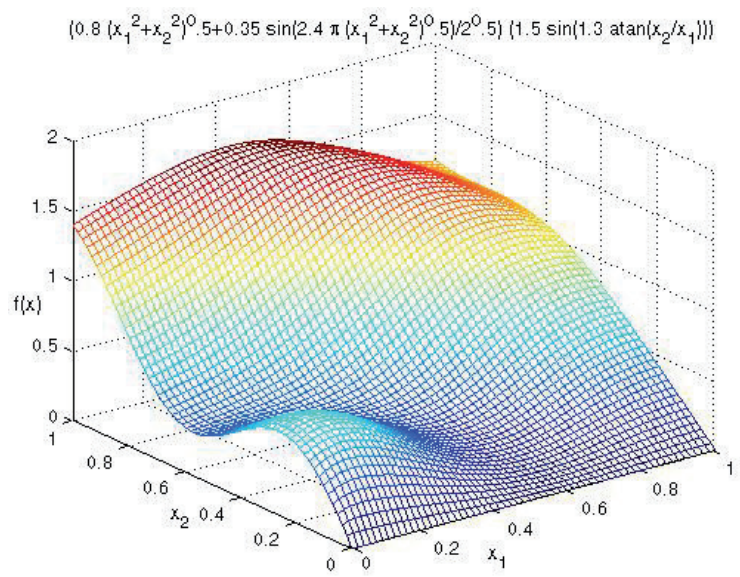

Fig. 4. Real function image of example 4.1.

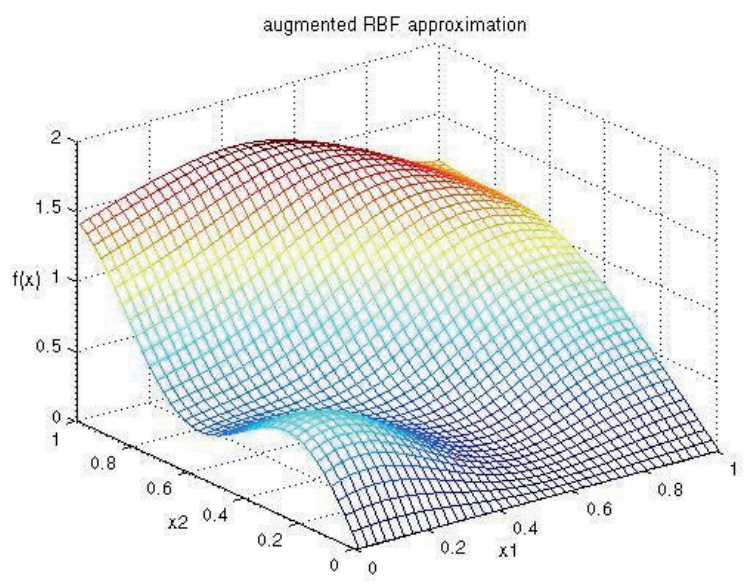

Fig. 5. Approximate function image of example 4.1.

Table 2. Comparison of the results of different algorithms.

\begin{tabular}{ccccc}
\hline \multirow{2}{*}{ Algorithm } & \multicolumn{2}{c}{ Optimal point Objective value } & Number of \\
& $x_{1}$ & $x_{2}$ & $f(x)$ & objective calculations \\
\hline GA & 0.4262 & 0.9999 & 1.6518 & 1304 \\
SAA & 0.4276 & 0.9999 & 1.6518 & 416 \\
AG-RBF-SQP & 0.4050 & 1.0000 & 1.6507 & 37 \\
\hline
\end{tabular}

In Table 2, the right column shows the number of objective calculations with different algorithms. We note that the present algorithm is very fast with respect to the two others. The convergence is attained with only two times of model establishment and 37 calls of objective calculations. The first model is established by using the 36 calls at the 36 chosen interpolating points; in the second model, the previously calculated objective functions and constraint functions at the 36 chosen points are kept and one more call is carried out at the optimal point obtained with the first model. We can use all these 37 points or 36 points by removing the nearest point from the optimal point of the first model to establish the second model. Two SQP calculations are needed for the first and second models, respectively.

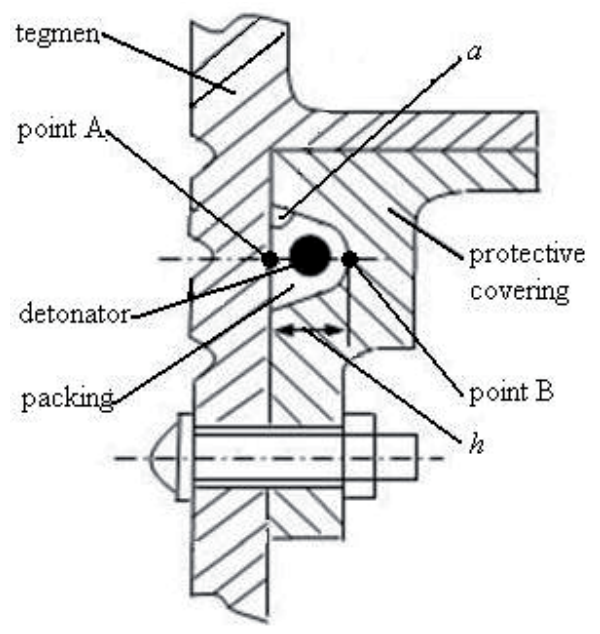

Fig. 6. 2D Section of axisymmetrical device for cutting off by explosion.

\subsection{Cavity shape optimization charged detonator of equipment cutting off by explosion}

An axisymmetrical device for cutting off by explosion is taken as an industrial application. Only its $2 \mathrm{D}$ section is illustrated (Fig. 6) and calculated. The cavity is charged with the detonator, so its shape and dimensions will determine the intensity and direction of the maximal cutting force. The cavity shape is defined by an arc and two segments, the height $h$ of the cavity and the obliquity $\alpha$ of the segments are taken as the design variables. For the initial design, $h$ is $0.36 \mathrm{~cm}, \alpha$ is $1.56 \mathrm{rad}$. The structure is divided into 1635 elements. The aim of the optimization is to give the maximal effective stress at the point $\mathrm{A}$ (element No. 1377) to cut of the tegmen and a effective stress inferior to the allowable limit $(310 \mathrm{MPa})$ at B (element No. 941) to preserve the protective covering. Therefore, the effective stress $\sigma_{\mathrm{EA}}$ at point $\mathrm{A}$ is taken as objective function and should be maximized, and the effective stress $\sigma_{\mathrm{EB}}$ at point $\mathrm{B}$ is taken as behaviour constraint. The following expression (16) describes this optimization problem:

$$
\begin{cases}\text { find } & \alpha, h \\ \max & \sigma_{\mathrm{EA}}(\alpha, h) \\ \text { s.t. } & \sigma_{\mathrm{EB}}(\alpha, h) \leqslant 310 \\ & 0.8 \leqslant \alpha \leqslant 1.56 \\ & 0.33 \leqslant h \leqslant 0.43\end{cases}
$$

Two similar schemas of interpolating points $(6 \times 6, a=$ $0.152, b=0.02$ in Fig. 2) and non interpolating points $(5 \times 5$ in Fig. 3$)$ are used for this example. At the 25 non-interpolate design points, the RMSEs of objective function and constraint condition obtained by using augmented RBF are 2.658 and 2.349 , respectively. They are a little better than those obtained by using RBF (2.734 and 2.408). Table 3 shows the comparison with SRSM-QP.

Similar to Example 4.1, this engineering application has two model establishing and two SQP calculations for every model. The AG-RBF-SQP algorithm use 37 structural analyses. From Table 3 and Figure 7 , we note that 
Table 3. Result comparison with SRSM-QP.

\begin{tabular}{cccccc}
\hline \multirow{2}{*}{ Algorithm } & \multicolumn{2}{c}{ Optimal point } & Objective value & Constraint value & Number of \\
& $\alpha /(\mathrm{rad})$ & $h /(\mathrm{cm})$ & $\sigma_{E A} / \mathrm{MPa}$ & $\sigma_{E B} / \mathrm{MPa}$ & structural analyses \\
\hline SRSM-QP [18] & 0.9914 & 0.3300 & 262.2200 & 296.5100 & 85 \\
AG-RBF-SQP & 1.2371 & 0.3300 & 314.8250 & 295.8280 & 37 \\
\hline
\end{tabular}

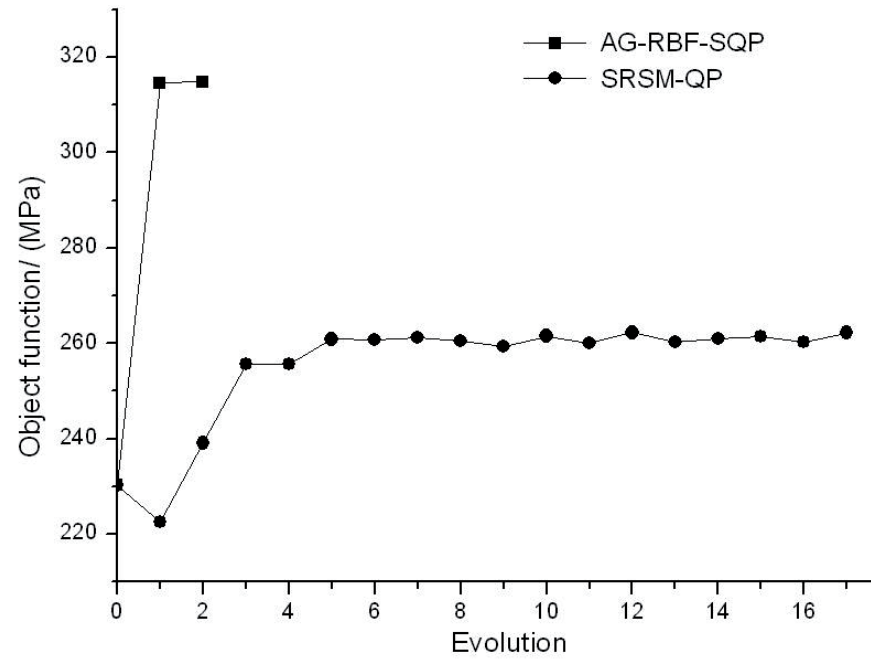

Fig. 7. Evolution of the objection function.

the global optimal objective value calculated by AG-RBFSQP is $20.1 \%$ larger than the local one by SRSM-QP, and the number of structural analyses is much smaller than that by SRSM-QP.

The simulated explosive process lasts $21 \mu \mathrm{s}$. Figure 8 shows the curves of the effective stresses versus time before and after optimization, the maximum effective stress at point $\mathrm{A}$ is $314.825 \mathrm{MPa}$ after optimization, it increase $36.7 \%$ compared with $230.31 \mathrm{MPa}$ before optimization, the maximum effective stress at point $\mathrm{B}$ changes very little, from 298.11 MPa before optimization to 295.828 $\mathrm{MPa}$ after optimization, it also satisfies the constraint.

\section{Conclusion}

In this paper, the augmented Gaussian Radial Basis Function is used to establish an approximate model for a global optimization procedure. This approximate model expressed explicitly in terms of the design variables allows to easily obtain the derivatives of the objective function and constraint functions, required in many optimisation algorithms. Taking into account the characteristics of the augmented Gaussian RBF, the method of Sequential Quadratic Programming (SQP) is adopted as optimisation solver. Since the derivatives of the objective function and constraint functions are calculated analytically without structural reanalyses, the present optimisation procedure is very fast. Furthermore, it does not require the user to provide any initial solution. The numerical tests show that the optimal results given by the present algorithm are very close to those obtained by the usually used GA

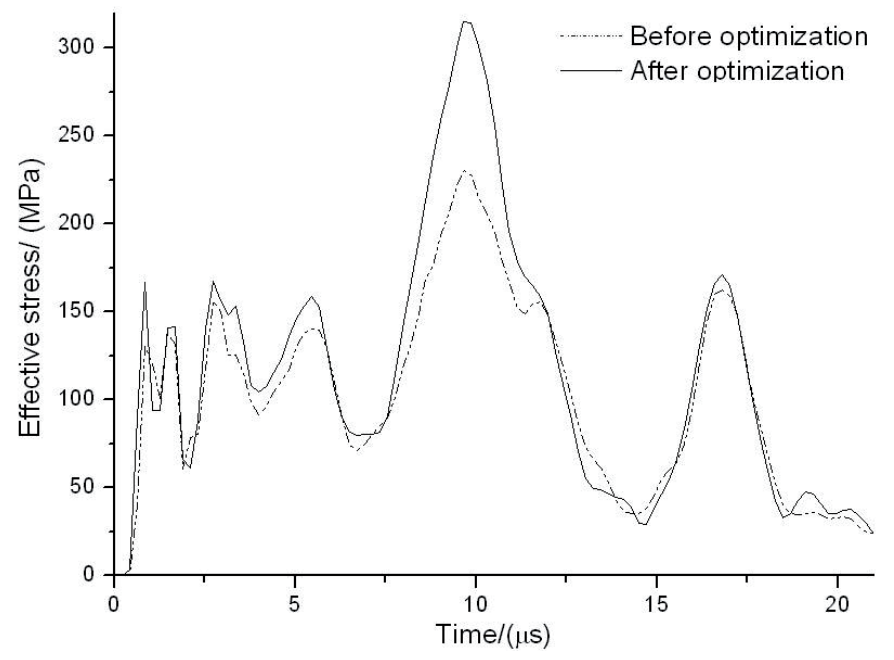

(a)

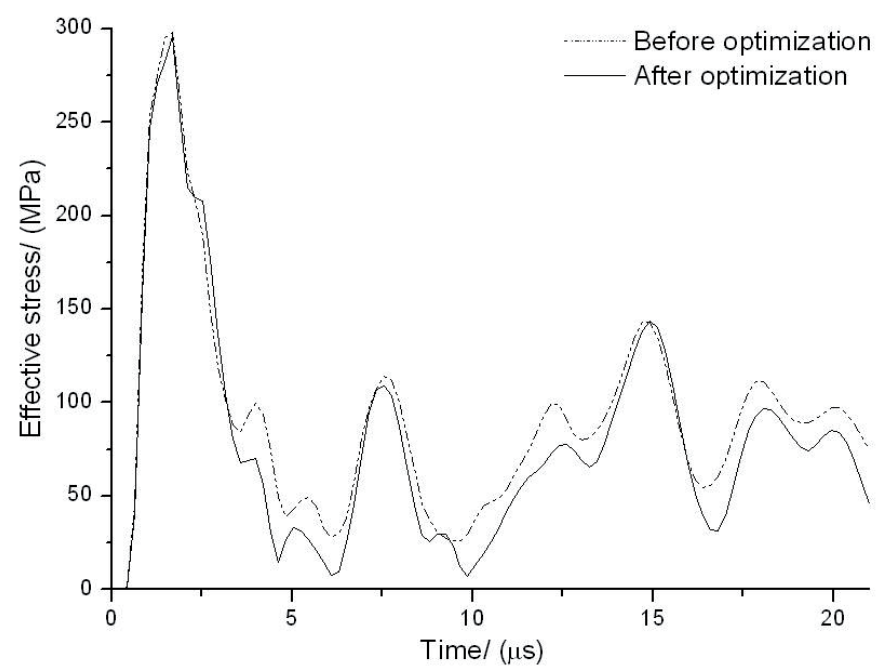

(b)

Fig. 8. Curves of the effective stress versus time. (a) Point A. (b) Point B.

and SAA, but it is much more efficient than these two optimisation algorithms and SRSM-QP algorithm.

\section{References}

1. L.A. Schmit, Structural design by systematic synthesis, in Proc. 2nd Nat. Conf. Elect. Comput. ASCE, 105-132 (1960)

2. L.A. Schimit, B. Farshi, Some approximation concepts for structural synthesis. AIAA J. 12, 692-699 (1974) 
3. R.H. Myers, D.C. Montgomery, Response Surface Methodology, Wiley and Sons New York (1995)

4. G. Venter, R.T. Haftka, Using response surface approximations in fuzzy set based design optimization. Struct. Multidisc. Optim. 18, 218-227 (1999)

5. S. Hosder, L.T. Watson, B. Grossman, W.H. Mason, H. Kim, H. RT, Steven EC, Polynomial Response Surface Approximations for the Multidisciplinary Design Optimization of a High Speed Civil Transport. Optimization and Engineering 2, 431-452 (2001)

6. T. Jansson, L. Nilsson, M. Redhe, Using Surrogate models and response surfaces in structural optimization-with application to crashworthiness design and sheet metal forming. Struct. Multidisc. Optim. 25, 129-140 (2003)

7. B. Liu, R.T. Haftka, L.T. Watson, Global-local structural optimization using response surfaces of local optimization margins. Struct. Multidisc. Optim. 27, 352-359 (2004)

8. Y. Zheng, P.K. Das, Improved response surface method and its application to stiffened plate reliability analysis. Engineering Structures 22, 544-551 (2000)

9. Y.K. Sui, S.P. Li, The application of improved RSM in shape optimization of two-dimension continuum, The International Conference on Computational Methods, December 15-17, (2004), Singapore

10. J.C. Mason, M.G. Cox, Radial basis functions for multivariable interpolation: a review in Algorithms for Approximation, Oxford University Press, Oxford (1987)
11. T. Krishnamurthy, Response surface approximation with augmented and compactly supported radial basis functions, in 44th AIAA/ASME/ASCE/AHS/ASC structures, structural dynamics, and materials conference, Norfolk, Virginia, April 7-10 (2003)

12. H.B. Fang, M.F. Horstemeyer, Global response approximation with radial basis functions. Eng. Optimiz. 38, 407-424 (2006)

13. Y.K. Sui, in Modeling, Transformation and Optimization: New Developments of Structural Synthesis Method, Dalian University of Technology Press (1996) (in Chinese)

14. Y.K. Sui, A.Q. Zhang, L.C. Long, Rational move limits and its application in optimization algorithm. J. Basic Science and Engineering (to be published, in Chinese)

15. G.N. Xuan, R.W. Cheng, X.J. Yu, G.G. Zhou, in Genetic algorithm and engineering optimization, Qinghua University Press Beijing, (2004) (in Chinese)

16. R.L. Yang, J.F. Gu, An efficient simulated annealing algorithm for global optimization. Systems EngineeringTheory Practice 17, 30-33 (1997) (in Chinese)

17. http://7d-soft.com/cn/download.htm

18. Z.G. Yang, M. Chen, Y.K. Sui, Application of response surface methodology in optimum design of cylindrical shell structure. J. Projectiles, Rockets, Missiles and Guidance (to be published, in Chinese) 